\title{
BGRP: Sink-Tree-Based Aggregation for Inter-Domain Reservations
}

\author{
Ping Pan \\ Bell Laboratories \\ Holmdel, NJ \\ pingpan@bell-labs.com
}

\author{
Ellen L. Hahne \\ Bell Laboratories \\ Murray Hill, NJ \\ hahne@bell-labs.com
}

\author{
Henning Schulzrinne \\ Columbia University \\ New York, NY \\ hgs@cs.columbia.edu
}

January 31, 2000

\begin{abstract}
Resource reservation must operate in an efficient and scalable fashion, to accommodate the rapid growth of the Internet. In this paper, we describe a distributed architecture for inter-domain aggregated resource reservation for unicast traffic. We also present an associated protocol, called the Border Gateway Reservation Protocol (BGRP), that scales well, in terms of message processing load, state storage and bandwidth. Each stub or transit domain may use its own intra-domain resource reservation protocol. BGRP builds a sink tree for each of the stub domains. Each sink tree aggregates bandwidth reservations from all data sources in the network. Since backbone routers maintain only the sink tree information, the total number of reservations at each router scales linearly with the number of Internet domains $N$. (Even aggregated versions of the current protocol RSVP have a reservation count that can grow like $O\left(N^{2}\right)$.) BGRP maintains these aggregated reservations using "soft state." To further reduce the protocol message traffic, routers may reserve bandwidth beyond the current load, so that some sources can join or leave the tree without sending messages all the way to the tree root. BGRP relies on Differentiated Services for data forwarding, hence the number of packet classifier entries is extremely small.
\end{abstract}

KEYWORDS: Resource reservation protocol. Aggregation. Sink Tree. Differentiated Services. Internet Quality of Service. RSVP.

Submitted to the Journal of Communications and Networking 


\section{Introduction}

Resource reservation was originally defined to support end-to-end QoS guarantees for a range of QoSsensitive applications, including multimedia-on-demand and teleconferencing [1]. Recently, Internet Service Providers (ISPs) have started to use the same reservation mechanisms to provide customer-level Virtual Private Networks (VPNs) [2] and to allocate network resources between Differentiated Service classes [3]. We believe that the applications of resource reservation and the demand for it will continue to grow, and that reserved QoS will eventually come to be seen as an indispensable feature of Internet service. At the present time, there are three challenges to the widespread use of reserved QoS: reservation protocol scalability, packet forwarding scalability, and inter-domain management.

Reservation protocol scalability. Resource reservation schemes must scale well with the rapidly growing size of the Internet. A router may be able to handle tens of thousands of simultaneous reservations [4], but not hundreds of thousands, and certainly not millions. Today's traffic volume is bad enough: as we will explain in Sec. 5.1, we have measured hundreds of thousands to millions of flows at the MAEWest network access point (Table 1); if many of these flows were to request resource reservations, the protocol overhead would swamp the router. But projected future traffic growth is an even more serious problem. The overhead of the current protocol RSVP [5,6] can grow like $O\left(N^{2}\right)$, where $N$ is the number of Internet end hosts. (RSVP must maintain separate routing state, called PATH state, for each reserved source-destination pair, in order to reverse-route RESV messages from destination to source.) Fig. 1 $[7,8,9]$ shows the growth of $N$ over the last six years, from 2 million to 60 million. This means that $N^{2}$ grew from $4 \cdot 10^{12}$ to $4 \cdot 10^{15}$ during that time! With no end in sight, $N^{2}$ is growing much faster than improvements in processing speeds or memory sizes. Therefore, we will have to find a reservation scheme that scales better than conventional RSVP. In this paper we will propose a protocol, called the Border Gateway Reservation Protocol (BGRP), that fixes this scaling problem in two ways. First, BGRP overhead scales linearly with the size of the Internet; i.e., $O\left(N^{2}\right)$ is reduced to $O(N)$. Second, BGRP uses "a smaller $N$ ". The overhead of the basic BGRP protocol is proportional to the number of Internet carrier

domains (also called Autonomous Systems (AS)), while an enhanced version of BGRP has overhead 
proportional to the number of IP networks (i.e., the number of announced IP address prefixes).

Data forwarding scalability. In addition to the overhead of the protocol that reserves QoS, another scaling issue is the overhead of the packet classifiers, enqueuers and schedulers that enforce the reserved QoS [10]. The current QoS architecture, called Integrated Services (IntServ) [1], requires backbone routers to classify and schedule packets on a per-flow basis. Our BGRP protocol, however, is designed to work within the newly proposed Differentiated Services (DiffServ) QoS architecture [11]. With DiffServ, all flow-related handling of packets (e.g., classification, policing, shaping) is done at the edges of the network. At the edge, packets are assigned to one of a few dozen QoS classes. Backbone routers queue and schedule packets according to their QoS class only.

Inter-domain adminstration. In general, Internet flows traverse several different network domains. Each ISP would prefer to manage its own network resources and enforce its own internal traffic engineering policies [12], acting as independently as possible of other carriers. Ideally, a domain should only have to reveal simple delivery commitments to its peering domains. There should be an inter-domain reservation system that uses these delivery commitments to establish a reservation path through multiple domains. Each domain would set up transit reservation flows using its own preferred intra-domain reservation mechanism. BGRP is specifically designed for such an environment. It operates on the boundaries between ISPs, leaving each ISP free to manage its own domain independently. Once QoS traffic management conforms itself to the technical and business "topology" of the Internet - a loosely coupled collection of competing and mistrustful carriers, barely cooperating through bilateral peer routing arrangements - then charging customers for QoS should become practical. We expect that the forging of this final missing link will encourage explosive deployment of QoS reservation mechanisms.

This paper is organized as follows. In Sec. 2, we quantify the amount of aggregation needed to make a resource reservation protocol that today's routers can comfortably handle. Then, in Sec. 3, we propose a reservation architecture for unicast traffic and a corresponding protocol BGRP that operate at this desired level of aggregation. The basic idea is to build a sink tree for each of the destination domains. Each sink tree aggregates reservations for flows from all data sources in the network to that destination domain. 
Sec. 4 describes various ways to improve the performance of the basic BGRP protocol. The scaling benefits of our approach are evaluated in Sec. 5. Recent work by others on the reservation scaling problem is briefly discussed in Sec. 6. We summarize our investigation and describe future work in Sec. 7.

\section{Aggregating Reservations}

How many simultaneous reservations can a router handle? In a recent study [4], we showed that a lowend router can set up 900 new RSVP reservations or refresh up to 1600 reservations per second, allowing it to sustain about 45,000 flows. To handle that many reservations, the router has to suspend routing computation and packet forwarding, due to hardware and CPU constraints. While backbone routers have more CPU power than the low-end router used for the measurements, other results [13] indicate that frequent routing computation due to route instability may already tax the CPU. Thus we, along with some other RSVP developers we have consulted, believe that in many networking environments, routers do not have enough CPU power to sustain hundreds of thousands of reservations.

If we assume a router can handle tens of thousands of reservations, but not hundreds of thousands, then what degree of aggregation is required? There are several ways to go about answering this question. In this section, we will give some ballpark estimates, based mostly on the data in Fig. 1. Much later, when we evaluate the performace of BGRP in Sec. 5.1, we will give more precise estimates, based on our statistical analyses of Internet packet traces.

Conventional RSVP reserves resources for each application-to-application flow. For simplicity, let us count host-to-host flows instead. According to Fig. 1, there are $36 \cdot 10^{14}$ source-host/destination-host pairs! This is enormously more than a router can handle, but of course, not all the host-to-host flows will be active at once, and not all active flows will pass through a given router or a given link. So let us try another upper bound on host-to-host flows that is somewhat more realistic: how many reserved flows of non-zero bandwidth can fit on a link of finite bandwidth? Let us assume that $16 \mathrm{~kb} / \mathrm{s}$ (enough, say, for a good quality packet voice call) is the finest granularity of reservation. Then an OC-192 (10 Gb/s) link might be called upon to support up to 600,000 reservations. This suggests that we may not be able to 
afford to reserve for individual host-to-host flows. Some aggregation of reservations is needed.

How might this aggregation be done? There are several ways to aggregate based on "regions" of IP addresses. A "region" can be defined at various granularities: e.g., one host, or one network, or one AS. The simplest option is to aggregate for each source-region/destination-region pair; i.e., on a given link, aggregate the reservations for all flows from a given source region to a given destination region. Alternatively, we could aggregate for each source region, i.e., on a given link, aggregate the reservations for all flows from a given source region to anywhere. Or we could aggregate for each destination region, i.e., on a given link, aggregate the reservations for all flows from anywhere to a given destination region.

Which of these aggregation options might be adequate in reducing the number of simultaneous reservations to a level that a router can handle? (There is no point in aggregating more than necessary, since this will make the protocol needlessly complex.) According to Fig. 1, there are approximately 6,000 AS, 60,000 networks, and $60,000,000$ hosts in the Internet today. That makes $36 \cdot 10^{6}$ AS pairs, $36 \cdot 10^{8}$ network pairs, and $36 \cdot 10^{14}$ host pairs. Comparing these six numbers to our estimate of router capacity determines our target degree of aggregation: one aggregate reservation for each region, where the size of a region is somewhere between one AS and one network. Furthermore, as we shall explain below, we prefer to produce one aggregate reservation for each destination region, rather than one per source region, because destination-based aggregates have a convenient tree structure.

Whether a simple hop-count metric is used or more sophisticated metrics are employed, most unicast routing algorithms determine shortest paths. In networks where the shortest paths are unique, the principle of optimality guarantees that the shortest paths to any destination form a tree; the shortest paths from any source are also guaranteed to form a tree. If there are multiple shortest-length paths, however, the existence of trees depends on the tie-breaking rules in the routing algorithm. The BGP routing algorithm [14] establishes "virtual edges" by using reachability as a definition for the existence of a link in the graph. BGP routes follow the shortest paths to the destinations. When there are multiple equal-length paths, BGP breaks ties in a way that guarantees sink trees, but not necessarily source trees. (Specifically, a BGP router forwards all packets to the same destination via a single next-hop router.) Since reservations are made 
along routes chosen by the routing algorithms, it is natural to aggregate these reservations along the sink trees formed by BGP routing. In Sec. 3, we present a new signaling protocol, called the Border Gateway Reservation Protocol (BGRP), that supports this architecture.

\section{The Border Gateway Reservation Protocol (BGRP)}

\subsection{Model and Terminology}

As shown in Fig. 2, the Internet is composed of a number of domains or autonomous systems (AS) that exchange user traffic among each other. A domain can be classified as either a $s t u b$ domain or a transit domain. Any path through a stub domain will either originate or terminate at a router in that domain; transit domains do not have this restriction. A domain connects to a number of other domains via border routers. We assume all border routers use BGP [14] for inter-domain routing. We define $\mathcal{R}=\left\{R_{1}, R_{2}, \ldots, R_{n}\right\}$ as the set of border routers in transit domains, $\mathcal{S}=\left\{S_{1}, S_{2}, \ldots, S_{m}\right\}$ as the set of border routers in stub domains, and $\mathcal{H}_{i}=\left\{h_{1}, h_{2}, \ldots, h_{j}\right\}$ as the set of end hosts in $\mathrm{AS}_{i}$. In this paper, inter-domain reservations

originate and terminate at routers in $\mathcal{S}$. We denote the direction of packets traveling from source towards sink as downstream, with upstream as the opposite direction. For simplicity, we assume in this paper that all active hosts are in stub domains. (In reality, a transit router $R_{i}$ could also play the role of a source or sink router for end users in its domain). Moreover, while in reality there are likely to be multiple routers in a domain between border routers, they do not participate in our inter-domain reservation protocol and are thus not shown in our figures.

\subsection{Overview of Protocol Operation}

The BGRP protocol operates only between border routers. We shall use the term BR-hop to denote the "virtual hop" between two "adjacent" border routers participating in BGRP. These "adjacent" border routers can be in different domains or in the same domain.

BGRP includes several control messages: PROBE, GRAFT, ERROR, REFRESH, and TEAR. To 
avoid delivery delay problems, BGRP messages are reliably delivered. PROBE and GRAFT messages specify reservation parameters such as traffic class and bandwidth. This paper assumes that bandwidth (for each traffic class) is the only reserved resource and that bandwidth reservations are additive. In practice, however, statistical multiplexing could be used, and other resources such as buffers could be reserved.

Reservation sources initiate PROBE messages to determine the network resource availability and the exact reservation path. Each PROBE message consist of a reservation request and destination network information. PROBE messages travel downstream from stub domain border routers. Border routers use BGP routing information and bilateral QoS agreements to forward PROBE messages. PROBE messages collect routing information along the reservation path, similarly to IP Record Route Option, but they do not install any reservation state or any routing state in the routers. (One might argue that QoS routing protocols would be sufficient for finding the path. In an inter-domain environment, however, we believe that the reservation path depends as much on ISP policy as on resource availability. Since each ISP only advertises its resource allocation policy to its immediately adjacent peers in the form of bilateral agreements, a resource user may have to actively probe the network to determine the edge-to-edge routing path for its reservation.)

Reservation sinks, upon receiving PROBE messages, return GRAFT messages to set up the appropriate reservations inside the network. The GRAFT message uses the previously collected routing information and traverses exactly the reverse path that the PROBE message took. Routers on the path keep one reservation entry for each sink tree. Among other things, this reservation entry identifies the root of the sink tree. Each reservation root is defined as the combination of the reservation destination information and the ID of the sink border router that processed and terminated the original PROBE message. (The sink border router ID is necessary in case the destination stub domain is multi-homed, because several of that domain's border routers could receive PROBE messages and thus become reservation sinks.) When processing a GRAFT message, each border router interfaces with intra-domain protocols to set up transit reservations within its domain. In case of reservation failures during probing or grafting, the routers send ERROR messages to inform the users. 
Reservation sources and sinks transmit PROBE and GRAFT messages only once during the lifetime of a reservation. BGRP reservations are maintained as "soft state", i.e., the border routers must periodically exchange REFRESH messages with their peers to keep their reservations "alive". If a border router does not hear an expected REFRESH for a reservation, it assumes a link failure, route change, or user termination and removes the reservation. The BGRP protocol also includes optional TEAR messages that routers can send to remove reservations in peer routers more quickly.

The BGRP protocol differs from RSVP in three important ways: stateless probing, reservation aggregation, and bundled refresh. Let us explain these differences. The RSVP PATH message installs routing state at intermediate routers, to guide the RSVP RESV message back to the data sender. Routers must therefore keep both sender and destination information. In a network with $N$ nodes, this may require $O\left(N^{2}\right)$ entries. BGRP's PROBE messages, however, install no state in the routers. (BGRP's GRAFT messages do store reservation information, but only $O(N)$ entries, because this information is per-sink, not per-source.) The second difference concerns the combining of reservations. RSVP can combine reservations in two ways. First, RSVP allows multiple (multicast) receivers to merge their reservations for the same sender (or set of senders) into a single reservation whose size is roughly the maximum of the individual reservations. Second, RSVP offers per-source and shared reservation styles; in the latter, multiple (multicast) senders take turns sharing a single reservation [15]. BGRP reservation aggregation is different from either of these. BGRP aggregates reservations from different (unicast) senders to the same receiver by adding them together. The final difference is that RSVP transmits PATH and RESV messages periodically to refresh each individual reservation separately, while BGRP bundles all reservation messages into one periodic refresh. (Similar enhancements have recently been proposed for RSVP itself [16].)

\subsection{Example}

Fig. 3 illustrates how BGRP works. Suppose $H_{1}$ in $A S_{1}$ needs to set up a reservation to $H_{5}$ in $A S_{5}$. $S_{1}$ sends a PROBE message containing the source ID $S_{1}$, the ID of the destination in $\mathcal{H}_{5}$ (either an application, a host or a subnet), the traffic class $C$, the bandwidth requirement $B W_{1,3}$, and an empty 
route record field. (In this example, $S_{1}$ launches a PROBE at the behest of a particular host. In a VPN application, however, $S_{1}$ could initiate a PROBE message toward $S_{3}$ directly, in an effort to set up a virtual "trunk" between their two domains.) When the PROBE message arrives at $R_{1}$, the router consults $A S_{3}$ 's resource database and the bilateral agreement between $A S_{1}$ and $A S_{3}$. If $A S_{3}$ can accept the requested reservation, $R_{1}$ inserts its own IP address into the route record field and forwards the PROBE message downstream. Otherwise, $R_{1}$ sends an ERROR message back to $S_{1}$. The selection of the downstream border router depends on intra-domain traffic engineering requirements [12] and BGP routing policy. In this example, $R_{1}$ forwards the message to $R_{3}$, as determined from the BGP NEXT_HOP path attribute. To prevent loops, each router checks whether the current route record already contains the router's own address. Assume that $R_{3}, R_{4}$ and $R_{5}$ all accept the reservation. When the PROBE message arrives at $S_{3}$, $S_{3}$ determines that the destination in $\mathcal{H}_{5}$ belongs to its local domain $A S_{5}$, so $S_{3}$ terminates the probing process. The final route record in the PROBE message is $\left(R_{1}, R_{3}, R_{4}, R_{5}\right)$.

Now $S_{3}$ sends a GRAFT message back toward $S_{1}$ to set up the desired reservation along the path. The GRAFT message is source-routed using information gathered in the route record of the PROBE message. The GRAFT message contains the traffic class $C$, bandwidth requirement $B W_{1,3}$, source ID $S_{1}$, route record $\left(R_{1}, R_{3}, R_{4}, R_{5}\right)$, sink ID $S_{3}$, and a tree ID label $L$. The tree ID label is assigned by the sink border router $S_{3}$. The label is used to uniquely identify a reservation tree, because there may be multiple reservation trees rooted at $S_{3}$. A tree ID label can be in the form of a CIDR prefix or the AS number. Assume that the reservation is successfully established between $S_{1}$ and $S_{3}$.

Now suppose that $S_{3}$ receives another PROBE for the same traffic class $C$, this one requesting bandwidth $B W_{2,3}$ from $S_{2}$ to $\mathcal{H}_{5}$. $S_{3}$ sends back a GRAFT message to $R_{5}$ containing traffic class $C$, bandwidth requirement $B W_{2,3}$, source ID $S_{2}$, route record $\left(R_{2}, R_{3}, R_{4}, R_{5}\right)$, sink ID $S_{3}$, and the same tree ID label $L$ used in the previous GRAFT message. $R_{5}$ recognizes this as an increment to the existing tree $L$ and increases the reserved bandwidth for class $C$ between $R_{5}$ and $S_{3}$ to $B W_{1,3}+B W_{2,3}$. Then $R_{5}$ forwards the GRAFT message to $R_{4}$, while using the intra-domain reservation protocols of $A S_{4}$ to update the internal reservation between $R_{4}$ and $R_{5}$. (Either $R_{5}$ or $R_{4}$ could be the initiator of this internal change, depending 
on the intra-domain reservation protocol.) The reserved bandwidth of class $C$ between $R_{4}$ and $R_{5}$ increases to $B W_{1,3}+B W_{2,3}$. Similarly, router $R_{4}$ forwards the GRAFT message to $R_{3}$ while incrementing the reservation between them. When the GRAFT arrives at $R_{3}$, that router creates a new reservation tree branch to $R_{2}$ with bandwidth $B W_{2,3}$. The reservation finishes when the GRAFT arrives at $S_{2}$. If any router $R_{i}$ cannot set up the new reservation, it sends an ERROR message back to the sink to inform it of the failure. Along the way, the ERROR message removes the reservation.

Router $R_{3}$ maintains the following state: sink tree ID $S_{3}$, tree label $L$, traffic class $C$, adjacent downstream border router $R_{4}$, bandwidth reserved to $R_{4}$ (viz., $B W_{1,3}+B W_{2,3}$ ), adjacent upstream border routers $R_{1}$ and $R_{2}$, and bandwidth reserved from each adjacent upstream border router (viz., $B W_{1,3}$ from $R_{1}$ and $B W_{2,3}$ from $R_{2}$ ).

\section{BGRP Enhancements}

The basic BGRP protocol aggregates reservations into trees, thereby reducing the number of reservations. We will quantify this in Sec. 5.1 and 5.2. Reducing the number of reservations obviously shrinks the memory needed to store the control state information. It also reduces the overhead associated with REFRESH messages for all these pieces of control state; refresh costs include CPU processing and link bandwidth. These savings take us much of the way toward our goal. However, BGRP's other control messages, PROBE and GRAFT, also consume processing and bandwidth. We would like to control the volume of these control messages as well and thereby add another dimension of scalability to BGRP. This can be done by making the following enhancements to the protocol.

Over-reservation, Quantization and Hysteresis. Leaf nodes, in their PROBE messages, can request more bandwidth between themselves and the tree root than is currently required. (One can think of this as aggregated advance reservations on behalf of unknown parties.) Nodes can also coarsely quantize the requested bandwidth, e.g., restrict it to multiples of some quantum $Q$. Hysteresis can also be employed; e.g., if the bandwidth requested by a leaf node has just jumped from $3 Q$ to $4 Q$ because its bandwidth requirement has just exceeded $3 Q$, then that leaf node should not reduce its request back to $3 Q$ until its 
bandwidth requirement drops below some threshold $T<3 Q$. These changes can dramatically reduce the volume of control messages, as we will quantify in Sec. 5.4.

CIDR Labeling and Quiet Grafting. Suppose that the branches of a BGRP sink tree are labelled with the CIDR prefix associated with the tree root. Then a router on that sink tree will be able to recognize whenever an incoming PROBE message "belongs" to that tree, viz., whenever the reservation destination belongs to that CIDR prefix. Also suppose that this tree node can over-reserve bandwidth between itself and the tree root. These two modifications enable a new tree operation called quiet grafting, whereby a new branch can be grafted onto an existing reservation sink tree without any PROBE or GRAFT messages being passed between the grafting node and the tree root. To demonstrate quiet grafting, let us construct the sink tree shown in Fig. 3. Suppose that initially $S_{1}$ requests 10 units of reserved bandwidth to $H_{5}$ in a PROBE message. Knowing that $H_{5}$ is a popular destination, $R_{3}$ inflates this request to 15 units as it processes the passing PROBE. When the GRAFT message returns from tree root $S_{3}$, it reserves 15 units at each BR-hop, until reaching $R_{3}$. Node $R_{3}$ deflates the amount to 10 units and passes the GRAFT back toward $R_{1}$ and $S_{1}$. Now 10 units of bandwidth have been reserved from $S_{1}$ to $R_{3}$, and 15 units have been reserved from $R_{3}$ to $S_{3}$. In $R_{3}$ 's own internal bookkeeping for these 15 units, $R_{3}$ considers 10 units as “belonging" to $R_{1}$ 's tree branch, and it considers 5 units as over-reserved. Now suppose $S_{2}$ requests 3 units of reserved bandwidth to $H_{5}$ in its PROBE message. When this PROBE reaches $R_{3}, R_{3}$ recognizes that it is already sitting on a sink tree to $H_{5}$ and that there is sufficient excess bandwidth already reserved between $R_{3}$ and $S_{3}$ to satisfy $S_{2}$ 's needs, so that $R_{3}$ can handle the new request directly itself, without propagating the PROBE further downstream. Therefore, $R_{3}$ terminates the PROBE, adjusts its internal bookkeeping to assign 3 of its 5 excess bandwidth units to this new tree branch, and launches a GRAFT message back toward $R_{2}$ and $S_{2}$. This GRAFT establishes a 3-unit reservation between leaf $S_{2}$ and grafting node $R_{3}$.

Self-Healing. When a route changes, BGRP has the option of moving the affected reservations to the new route, without demolishing the entire reservation tree and re-creating the tree from scratch. Assume that the reservation tree is labeled with the destination CIDR prefixes, as described above. When a tree node detects a route change, it can initiate a new PROBE toward the sink. When this PROBE reaches 
a downstream router on the stable part of the old reservation tree, that router can respond with a GRAT and thus repair the part of the reservation between the two routers. We call this process self-healing.

Reservation Damping. Labovitz et al. $[13,17]$ have shown that, of three million BGP route changes each day, 99\% were pathological and did not reflect real network topological changes. If routers make BGRP reservation changes in response to every route change, there could be a high volume of nearly worthless reservation messages in the network. On the other hand, if the routers do not move a reservation, and the route change turns out to be legitimate and stable, then the data will have lost its reservation. This is the trade-off in deciding when to adjust reservations. Here, we propose a damping function for BGRP. The goal of reservation damping is to delay the initiation of the self-healing process until the changing routes have stabilized. The delay depends on the probability of future instability of the route. Routes that change frequently will be delayed longer. Similar to $[18,19]$, we propose an exponential function $\tau=(1+\Delta)^{n} \cdot T$ for computing the delay $\tau$ between PROBEs sent due to route changes. $\Delta$ and $T$ are the parameters to adjust the damping, and $n$ is the number of route changes measured in a time interval.

\section{Protocol Scaling Evaluation}

\subsection{Estimating Reservation Volume from Packet Traces}

We would like to determine how well in practice BGRP will reduce the volume of reservations, as compared with conventional RSVP and its aggregated region-to-region extensions. To that end, we examined a 90-second traffic trace from the MAE-West network access point (NAP). ${ }^{1}$ We categorized about 3 million IP packet headers according to their transport-layer port, IP address, IP network prefix, and AS. Table 1 shows the results. Suppose that all traffic desired a reservation of some quality level. If we use conventional RSVP and reserve for each source-destination pair at the application level, then the total number of active reservations would be 339,245. This data strengthens an estimate we made in Sec. 2, viz., that

\footnotetext{
${ }^{1}$ The 90-second traces date from June 1, 1999; see [20]. The AS information was collected on June 10, 1999 and analyzed by Sean McCreary of NLANR/CAIDA.
} 
there can be hundreds of thousands of flows on a link, more than a router can handle, and hence some aggregation is necessary. Table 1 also shows that if we use aggregated versions of RSVP to reserve for source-destination pairs of various granularities, we can greatly reduce the number of flows. For example, for AS-to-AS aggregation, the number of flows in the 90 -second window was only 20,857 . If twenty thousand were as bad as things could get, then a backbone router would be able to handle one RSVP reservation for each source-destination AS pair. However, this number may be artificially low due to the small 90-second window. Let us see what happens if we observe over a month-long window. The last line of Table 1 shows AS counts seen at MAE-West during May 1999. ${ }^{2}$ During this month, MAE-West saw 5,001 destination AS; according to Fig. 1, this is essentially the complete AS roster. The number of different source-destination AS pairs viewed that month was enormous: 7,900,362 AS pairs, about a third of the 25 million possible combinations. Thus, unless routers tear down AS-to-AS reservations frequently, there may be too many such AS-level "trunks" to sustain in backbone routers. This data strengthens another estimate we made in Sec. 2, viz., that there can be millions of AS-to-AS flows, more than a router can handle, and hence the point-to-point aggregation style of RSVP is inadequate, even if each "point" is enlarged into a region the size of an AS. What is needed instead is BGRP's style of aggregation, with one reservation per destination AS, or at most one per destination network. The last column of Table 1 shows the gain of BGRP over RSVP, i.e., the ratio of RSVP flows to BGRP flows. The gain is greatest if the aggregation regions are large and if the reservations last a long time. At the AS level of granularity, for a one-month time duration, it is clear that the " $N$-vs- $N^{2}$ " problem in theory becomes an " $N$-vs- $N^{2}$ " problem in reality. Under these circumstances, BGRP outperforms RSVP by a factor of 1580.

Our assumption above was that every flow would request a reservation. Let us explore this issue further. Conventional wisdom holds that long-lived high-volume real-time applications like packet voice and packet video will want resource reservation. On the other hand, recent IETF proposals have suggested using resource reservation protocols to set up VPN links [2] and to provide service differentiation among users [3]. These reservations can be for scheduling priority as well as bandwidth. The bandwidth of an

\footnotetext{
${ }^{2}$ Analysis provided by Sean McCreary of NLANR/CAIDA.
} 
individual VPN flow or Differentiated Service flow might not be as large as a typical real-time flow, hence there might be even more such reservations on a given backbone link. To see whether BGRP shows the same gains over RSVP for both the voice/video and VPN/DiffServ models of reserved flows, we sorted the flows in our packet traces by size. We collected eight packet header traces from MAE West. The traces were collected three hours apart on June 1, 1999 [20]. Each trace comprises three minutes of all traffic at the NAP and contains about 33 million packet entries. We computed the number of bytes for each destination network address prefix (for BGRP-style reservations), and we also computed the number of bytes for each pair of source and destination prefixes (for RSVP-style reservations). We sorted the data into five categories: fewer than 50 b/s, 50-500 b/s, 500-2000 b/s, 2000-8000 b/s, and greater than 8000 b/s. Fig. 4 plots the distribution of flows by bandwidth, and it also plots the gain (i.e., the ratio of RSVP flows to BGRP flows) for each bandwidth class. Most of packets belong to the small-flow category (63.5\% for RSVP and 46.2\% for BGRP). Only 3621 (3.5\%) of the source-destination pairs and $1296(10.9 \%)$ of the destinations have an average bit rate over $2000 \mathrm{~b} / \mathrm{s}$. (Interestingly, there are more above- $8000 \mathrm{~b} / \mathrm{s}$ destination-only flows (719) than source-destination flows (516).) To summarize Fig. 4, if reservations are made only for high-volume sessions, then such flows are rare enough that RSVP scalability is not an issue, and using BGRP would not significantly reduce the number of such flows anyway. However, with VPN and Differentiated Services, where even the hordes of small flows may require QoS, RSVP scalability could be a problem and BGRP could be quite beneficial.

\subsection{Topological Distribution of Demand}

We use the simple model in Fig. 5 to compare the scaling properties of BGRP and RSVP. The model depicts a progression of domains along an Internet path, with access networks toward the left and right and backbone networks near the middle of the topology. We define $D$ as the maximum edge-to-edge distance, measured as the number of AS. A "node" $n_{i}$ in the figure represents an inter-domain traffic exchange point, which can be either a Point of Presence (POP) or a NAP. (In the real network from which this model is abstracted, each node $n_{i}$ could actually contain many routers and interconnect many AS.) 
A "link" $l_{i}$ in the figure represents an aggregation of all the real links that transport traffic from domain $i$ to domain $i+1$. The model also includes a reverse-directed link from $i+1$ to $i$, which is not shown in the figure. In addition to the diameter $D$, our model is characterized by the quantities $s_{i}$ and $d_{i}: s_{i}$ is the number of inter-domain reservation sources coming into $n_{i}$, and $d_{i}$ is the number of reservations sinks reached through $n_{i}$, not including those that $n_{i}$ reaches via $l_{i}$. In this model, the number of RSVP flows (i.e., source-destination pairs) on the uni-directional link $l_{i}$ is given by $L_{i}^{\mathrm{RVP}}=\sum_{j=0}^{i} s_{j} \sum_{k=i+1}^{D} d_{k}$, and the number of BGRP flows (i.e., one per destination) on $l_{i}$ is given by $L_{i}^{\text {BGRP }}=\sum_{k=i+1}^{D} d_{k}$. Node $n_{i}$ handles traffic in both directions, so the number of reservation flows traversing $n_{i}$ for the respective protocols is given by $\quad N_{i}^{\mathrm{RSVP}}=\sum_{j=0}^{i} s_{j} \sum_{k=i}^{D} d_{k}+\sum_{j=i}^{D} s_{j} \sum_{k=0}^{i} d_{k}-s_{i} d_{i} \quad$ and $\quad N_{i}^{\mathrm{BGRP}}=\sum_{j=0}^{D} d_{j}$.

We now study both RSVP and BGRP in different networking scenarios, computing the number of flows and associated gains. We set $D=9$, which is the maximum AS path length in the Internet today [21]. We simulated models with a total of 100 source and sink border routers. We assume that every source border router desires to set up a reservation to every sink border router. Three different demand distributions were constructed. For the Flat Topology, we set the number of reservation sources and sinks to be identical at each $n_{i}$; i.e., $s_{i}=d_{i}=5$, for $0 \leq i \leq 9$. For the Hierarchical Topology, we set $s_{i}=d_{i}=11$, for $3 \leq i \leq 6$, and $s_{i}=d_{i}=1$, for all other $i$. This models a hierarchical network where stub domains only contribute a small portion of the overall reservation flows, while most of the reservations are present at a few core transit domains. For the Selected Source Topology, we set all $s_{i}$ to 1 , and all $d_{i}$ to 9 . This reflects a network where the number of data sources is small, but the number of sinks (data receivers) is large. This is a typical scenario for web applications.

The results are shown in Fig. 6. Not surprisingly, BGRP maintains fewer reservations than RSVP. Fig. 6(c) shows that the largest gain occurs in the center of the network. Also, Fig. 6(b) shows that for RSVP, the number of reservations at a node depends on the node's topological location, whereas for BGRP, every node has the same number of reservations. 


\subsection{Reservation Dynamics}

We now consider the effect of reservation dynamics in our performance analysis. For both RSVP and BGRP, we shall determine the control state overhead and the control message overhead as functions of the arrival rate of individual flows, the mean lifetime of a flow, and the protocol refresh interval. We assume that reservations are explicitly torn down when no longer needed, and that the blocking rate for reservations is negligible for our purposes.

Recall that the "virtual hop" between two "adjacent" border routers is called a BR-hop. We define the sequence of border routers visited by an end-to-end traffic flow as an edge-to-edge BR-path. For RSVP, each BR-path establishes its own reservation. Let $F$ be the total number of BR-paths crossing a given border router $R_{i}$. For BGRP, these paths are aggregated into trees. Let $T$ be the total number of sink trees formed by the paths through $R_{i}$. To keep this analysis simple, we assume that each sink tree at $R_{i}$ is aggregating the same number $f=F / T$ of BR-paths.

Now let us model the individual end-to-end flows desiring reservations. Any number of these reserved flows may be multiplexed onto a given BR-path. We assume that the individual reserved flows for one path arrive in a Poisson stream of rate $\lambda$, and that the flow reservation lifetimes are exponentially distributed with mean $1 / \mu$. Then the number of individual flows on one BR-path is Poisson distributed with mean $\rho=\lambda / \mu$, and the number of individual flows on any given sink tree is Poisson distributed, with mean $\rho \cdot f$.

Let us determine the reservation counts of the protocols. In case of RSVP, the probability that the BR-path has a reservation, i.e., that at least one individual flow is reserved, is $1-e^{-\rho}$. Therefore, the number of reserved BR-paths at $R_{i}$ is binomially distributed with mean $\left(1-e^{-\rho}\right) \cdot F$. This is the average number of RSVP reservations for $R_{i}$. For large $\rho$, this approaches $F$, while for small $\rho$, this approaches 0. Now let us determine the BGRP reservation count at $R_{i}$. The probability that a given sink tree has a reservation at $R_{i}$, i.e., that at least one individual flow on at least one BR-path on that tree has requested a reservation, is $1-e^{-\rho \cdot f}$. The number of reserved trees on $R_{i}$ is thus binomially distributed with mean $\left(1-e^{-\rho \cdot f}\right) \cdot T=\left(1-e^{-\rho \cdot F / T}\right) \cdot T$. This is the average number of BGRP reservations for $R_{i}$. For large $\rho$, this approaches $T$, while for small $\rho$, this approaches 0 . We conclude that the reservation count advantage 
of BGRP with respect to RSVP is more pronounced when $\rho$ is large, where this gain approaches $F / T$. Fig. 7 shows the mean number of simultaneous reservations for BGRP and RSVP as $\rho$ ranges from 0.001 to 10 ; here we assume $F=100,000$ and $T=1,000$. For instance, when $\rho=1$, the gain is 63 . When $\rho=10$, the gain has essentially reached its maxiumum value of 100 .

Next we will compare the message rates for the two protocols. We will tally the control messages associated with reservations on one given unidirectional BR-hop. (Note that some of these "associated" messages actually travel on the reverse BR-hop.) When a new individual end-to-end flow for the given BR-hop is born, this counts as two messages in either protocol: PATH + RESV for RSVP, or PROBE + GRAFT for BGRP. Removing the reservation takes a single TEAR message, in either protocol. Since refresh is bidirectional, in both protocols, we count the refreshing of one reservation on a given BR-hop as two units of control message processing. (Refreshes for multiple reservations on the same BR-hop are assumed to be processed separately, even though they may be transmitted in a bundle.) Let $\eta$ be the refresh rate. For RSVP, the average message rate for each BR-path on the given BR-hop is $3 \lambda+2 \eta \cdot\left(1-e^{-\rho}\right)$. Hence, the average RSVP message rate for the BR-hop is $\left[3 \lambda+2 \eta \cdot\left(1-e^{-\rho}\right)\right] \cdot F$. For BGRP, the average message rate for one sink tree on the given BR-hop is $3 \lambda \cdot f+2 \eta \cdot\left(1-e^{-\rho \cdot f}\right)$. Hence, the average BGRP message rate for the BR-hop is $\left[3 \lambda \cdot f+2 \eta \cdot\left(1-e^{-\rho \cdot f}\right)\right] \cdot T$, which equals $3 \lambda \cdot F+2 \eta \cdot T \cdot\left(1-e^{-\rho \cdot F / T}\right)$. If $\lambda$ is much larger than $\eta$, then BGRP's PROBE, GRAFT and TEAR activities dominate its REFRESHes, and RSVP's initial PATH and RESV messages dominate their refreshed versions. In this case, RSVP and BGRP have the same message rates. (Fortunately, the over-reservation techniques that we proposed in Sec. 4 can dramatically reduce BGRP's message processing overhead at this end of the spectrum; we will analyze these improvements in Sec. 5.4 below.) On the other hand, if $\eta$ is much larger than $\lambda$, so that REFRESH activity dominates, and if $\rho$ is large, then BGRP does better than RSVP by a factor of $F / T$. Fig. 8 shows the average message rates per second, for BGRP and RSVP, as $\eta$ ranges from 0.0003 to 3.0 refreshes per second; here we assume $\lambda=0.001$ flows per second, $F=100,000, T=1,000$, and $\rho=10$. For instance, when $\eta=0.03$ (i.e., when the refresh interval is about 30 seconds), then the gain (i.e., the ratio of RSVP messages to BGRP messages) is 18 . When $\eta=3.0$, the gain is 95.8 . 


\subsection{Over-reservation, Quantization and Hysteresis}

Sec. 5.3 showed that BGRP processes fewer messages than RSVP, provided that most messages are REFRESHes rather than PROBEs, GRAFTs, and TEARs. Now we show how hysteresis can be used to curb the PROBE, GRAFT and TEAR activity. These savings come at a cost of some wasted reservable bandwidth, because the aggregate reservation sometimes exceeds the sum of the component flow requests.

We model a single aggregate reservation on a single BR-hop. We assume that the blocking rate for reservations is negligible for the purpose of measuring mean message rates. Any number of end-to-end flow reservations can be multiplexed into this aggregate. Assume that reserved flows arrive in a Poisson stream of rate $\lambda$ and that the flow reservation lifetimes are exponentially distributed with mean $1 / \mu$. Then the number of individual flows in our aggregate reservation is Poisson distributed with mean $\rho=\lambda / \mu$.

Assume that each individual flow requires one unit of bandwidth. We constrain the aggregate reservation to be a multiple of some quantum size $Q \geq 2$. Whenever the aggregate reservation is $k \cdot Q$ units, and this is just barely enough to satisfy the current individual flows, and a new individual flow reservation is requested, then the aggregate reservation jumps to $(k+1) \cdot Q$. This new quantum will only be relinquished when the sum of the individual flow requests drops to $(k-1) \cdot Q+1$. The amount of reservable bandwidth wasted due to over-reservation by this technique is less than $2 Q$ units.

We can model this system as a two-dimensional Markov process with state $(x, y)$, where $x$ is the number of currently reserved individual flows, and $y$ is the current aggregate reservation. Fig. 9 shows the state transition diagram for $Q=3$. The valid states are: state $(0,0)$, which we will ignore because it is transient; states $(x, Q)$ with $0 \leq x \leq Q$; and states $(x, k \cdot Q)$ with $k \geq 2$ and $(k-2) \cdot Q+2 \leq x \leq$ $k \cdot Q$. The structure of the state transition diagram makes it straightforward to determine the steady-state

probabilities. For most values of $x$, there are two possible values of $y$, i.e., two states. However, certain special values of $x$ have only one possible $y$ value, i.e., one state. These special values are: $x=0$, for which $y$ must equal $Q$, and $x=1, Q+1,2 Q+1,3 Q+1, \ldots$, for which the respective values of y must be $Q, 2 Q, 3 Q, 4 Q, \ldots$. Therefore, at these special points, the joint probability distribution $\pi(x, y)$ matches the marginal distribution of $x$ by itself, which is Poisson. It is straightforward to determine the probabilities 
of all the other states in terms of these special states. For $k \geq 1$ and for $(k-1) Q+2 \leq x \leq k Q$ :

$$
\begin{aligned}
& \pi(0, Q)=\quad e^{-\rho} \quad \pi(x, k \cdot Q)=\frac{e^{-\rho} \cdot \rho^{x} \cdot \sum_{i=0}^{k \cdot Q-x}\left[\rho^{i} \cdot(k \cdot Q-i) !\right]}{x ! \cdot \sum_{i=0}^{Q-1}\left[\rho^{i}(k \cdot Q-i) !\right]} \\
& \pi(((k-1) Q+1), k Q)=\frac{e^{-\rho} \cdot \rho^{((k-1) \cdot Q+1)}}{((k-1) \cdot Q+1) !} \quad \pi(x,(k+1) Q)=\frac{e^{-\rho} \cdot \rho^{x} \cdot \sum_{i=k \cdot Q+1-x}^{Q-1}\left[\rho^{i} \cdot(k \cdot Q-i) !\right]}{x ! \cdot \sum_{i=0}^{Q-1}\left[\rho^{i} \cdot(k \cdot Q-i) !\right]}
\end{aligned}
$$

The rate of control messages (PROBEs + GRAFTs + TEARs $)$ is: $3 \lambda \cdot e^{-\rho} \cdot \sum_{k=1}^{\infty}\left(\frac{\rho^{(k \cdot Q)}}{\sum_{i=0}^{Q-1}\left[\rho^{i} \cdot(k \cdot Q-i) !\right]}\right)$. This compares very favorably to the message rate $3 \lambda$ without hysteresis. Fig. 10 shows the message rate reduction factor for various values of $Q$ and $\rho$. For example, if $Q=10$ and $\rho=100$, then quantization and hysteresis reduce the BGRP message rate by a factor of about 100. (The curves in Fig. 10, while roughly decreasing, are not strictly monotonic. The ripples in the plots are due to the many "corners" in the state transition diagram of Fig. 9. For a given quantum size $Q$, as $\rho$ increases, the bulk of the probability mass zigzags upward and to the right through the state space. Since vertical state transitions produce protocol messages while horizontal transitions do not, these zigs and zags around the corners can produce ripples in the messaging efficiency plot.)

The analysis above dealt with a single aggregate reservation on a single BR-hop. It is a good model for a system where the leaves of sink trees are the only tree nodes that can initiate an over-reservation. However, if any tree node can initiate an over-reservation, and if the quiet grafting described in Sec. 4 is done, then additional savings in message rate are possible. Of course, an over-reserving router must recognize the future traffic on whose behalf the over-reservation was made, without sending a new PROBE message. For tree leaves that over-reserve, simple caching of the destination network IDs associated with each tree may be sufficient. For more complex situations, see the CIDR labeling discussion in Sec. 4.

\section{Related Work}

Recently, several authors have addressed scalable resource reservation, using either a server-based or a router-based approach. In server-based approaches, each domain has a bandwidth broker (or agent) which 
is responsible for selecting and setting up the aggregated reservation sessions. This approach has the advantage of removing the message processing and storage burden from routers. However, synchronizing reservation information among the bandwidth brokers and the routers may be complex. No aggregation takes place, so that each broker still has to deal with the requests of individual flows. Also, care must be taken so that the broker does not become a single point of failure for the domain. Variations of the server-based approach have been described in [11], [22], [23], and [24]. The latter proposal suggests a two-tier system where, within each domain, hosts use intra-domain reservation protocols such as RSVP to set up reserved flows. Inter-domain reservation protocols set up coarsely-measured reserved flows between domains. However, the proposal leaves the actual mechanism undefined. Alternatively, routerbased approaches that modify RSVP to support scalable reservation have been proposed in [25], [26] and [27]. (LSP tunnels [25] are designed to support intra-domain traffic engineering, but may also be used to set up trunks crossing multiple domains.) These proposals aggregate per-application reservation requests into reservation "trunks" between pairs of domains, by modifying sender template and session objects in RSVP to carry address and mask (“CIDR blocks”) or autonomous system (AS) numbers instead of 5-tuples (sender IP address, sender port, receiver IP address, receiver port, protocol). However, this

implies that routers in the backbone may have to maintain reservation state proportional to the square of the number of CIDR blocks or autonomous systems. Since the number of AS is currently about 6,000, the number of AS pairs exceeds 36,000,000. As we argued in Sec. 2 and 5.1, this is excessive. A more aggregated reservation scheme is needed. Finally, we mention the recent Boomerang protocol [28], in which end users send reservation requests and refresh messages to set up and maintain reservations. No per-flow state is stored at routers. However, the scalability of the control message processing is an issue.

\section{Conclusions, Future Work, and Acknowledgements}

This paper presented a distributed architecture and the BGRP protocol for inter-domain resource reservation, in which reservations are aggregated along sink trees. Our proposal relies on DiffServ for scalability of packet forwarding operations. The BGRP protocol scales well in terms of control state, message pro- 
cessing, and bandwidth. BGRP reduces control state by aggregating reservations; this reduces their number, and thereby reduces the memory needed to store the control information. Control message processing is the most important scalability issue. The cost of processing reservation messages depends on the volume of requests for new reservations, the volume of existing reservations requiring periodic refreshing, and the refresh frequency. BGRP economizes on all of these components. First, when we allow routers to over-reserve bandwidth with BGRP, then small reservations can join and leave the reservation sink tree without disturbing the entire tree. This reduces the volume of reservation set-up messages. Second, by aggregating reservations, BGRP reduces their number, and this proportionally reduces the refresh processing burden. Third, BGRP needs less frequent refreshes than does RSVP, for the following reason. RSVP control messages are unreliable and thus must be repeated at about three times the state-timeout interval, while BGRP refresh messages are transferred reliably hop-by-hop. The mechanisms described above reduce the number of control messages. Not only does this reduce the burden on the routers to process these messages, it also reduces the link bandwidth cost to transmit these messages.

This paper presented the basic architecture, protocol design and performance analysis. In the future, we will continue to explore various BGRP enhancements, such as reservation route pin-down, and to examine various options for rooting and labeling the reservation sink trees. We will also consider several options for PROBE message processing: whether resource availability really needs to be checked during this first protocol phase, and if so, whether the resource should also be reserved at that time. More dramatic changes, such as the use of source trees (instead of sink trees) and support for multicast, may also be considered. At the same time, we are implementing BGRP on backbone router platforms and will test it in realistic networking environments. We have proposed BGRP to the IETF [29].

We would like to thank Sean McCreary for helping us analyze the AS traces. Craig Labovitz advised us on various aspects of network operations that are relevant to BGRP. Tony Przygienda and Rohit Dube commented on the interaction between BGP and routing aggregation. We also thank Fred Baker, Kimberly Claffy, Der-Hwa Gan, George Swallow, and Andreas Terzis for many discussions leading to this work. 


\section{References}

[1] R. Braden, D. Clark, and S. Shenker, "Integrated services in the internet architecture: an overview," Request for Comments (Informational) 1633, Internet Engineering Task Force, June 1994.

[2] T. Li and Y. Rekhter, "A provider architecture for differentiated services and traffic engineering (PASTE)," Request for Comments (Informational) 2430, Internet Engineering Task Force, Oct. 1998.

[3] K. Nichols, V. Jacobson, and L. Zhang, "A two-bit differentiated services architecture for the internet," Internet Draft, Internet Engineering Task Force, May 1999. Work in progress.

[4] P. Pan and H. Schulzrinne, "YESSIR: a simple reservation mechanism for the Internet," ACM Computer Communication Review, vol. 29, pp. 89-101, Apr. 1999.

[5] L. Zhang, S. Deering, D. Estrin, S. Shenker, and D. Zappala, "RSVP: a new resource ReSerVation protocol," IEEE Network, vol. 7, pp. 8-18, Sept. 1993.

[6] R. Braden, Ed., L. Zhang, S. Berson, S. Herzog, and S. Jamin, "Resource ReSerVation protocol (RSVP) version 1 functional specification," Request for Comments (Proposed Standard) 2205, Internet Engineering Task Force, Sept. 1997.

[7] Internet Software Consortium, "Internet domain survey." http://www.isc.org/ds/.

[8] Telstra Network, “Telstra internet network performance reports.” http://www.telstra.net/ops/.

[9] T. Bates, “The cidr report.” http://www.employees.org/tbates/cidr-report.html.

[10] T. cker Chiueh, A. Neogi, and P. Stirpe, "Performance analysis of an RSVP-capable router," in Proc. of 4th Real-Time Technology and Applications Symposium, (Denver, Colorado), June 1998.

[11] S. Blake, D. Black, M. Carlson, E. Davies, Z. Wang, and W. Weiss, "An architecture for differentiated service," Request for Comments (Informational) 2475, Internet Engineering Task Force, Dec. 1998.

[12] J. Agogbua, D. Awduche, J. Malcolm, J. McManus, and M. O’ Dell, "Requirements for traffic engineering over MPLS," Internet Draft, Internet Engineering Task Force, June 1999. Work in progress.

[13] C. Labovitz, G. R. Malan, and F. Jahanian, "Internet routing instability," in SIGCOMM Symposium on Communications Architectures and Protocols, (Cannes, France), Sept. 1997.

[14] Y. Rekhter and T. Li, "A border gateway protocol 4 (BGP-4)," Request for Comments (Draft Standard) 1771, Internet Engineering Task Force, Mar. 1995.

[15] D. J. Mitzel and S. Shenker, "Asymptotic resource consumption in multicast reservation styles," in SIGCOMM Symposium on Communications Architectures and Protocols, (London, UK), pp. 226-233, Sept. 1994.

[16] L. Berger, D. Gan, G. Swallow, and P. Pan, "RSVP refresh reduction extensions," Internet Draft, Internet Engineering Task Force, July 1999. Work in progress.

[17] C. Labovitz, G. Malan, and F. Jahanian, "Origins of internet routing instability," in Proceedings of the Conference on Computer Communications (IEEE Infocom), (New York), Mar. 1999.

[18] P. Pan and H. Schulzrinne, "Staged refresh timers for RSVP," in Proceedings of Global Internet, (Phoenix, Arizona), Nov. 1997. also IBM Research Technical Report TC20966. 
[19] C. Villamizar, R. Chandra, and R. Govindan, "BGP route flap damping," Request for Comments (Proposed Standard) 2439, Internet Engineering Task Force, Nov. 1998.

[20] NLANR, "Nlanr network traffic packet header traces." http://moat.nlanr.net/Traces/.

[21] NLANR, "Nlanr as path lengths." http://moat.nlanr.net/ASPL/.

[22] O. Schelen and S. Pink, "Aggregating resource reservation over multiple routing domains," in Proc. of Fifth IFIP International Workshop on Quality of Service (IwQOS), (Cambridge, England), June 1998.

[23] S. Berson and S. Vincent, "Aggregation of internet integrated services state," in submitted to IWQOS, 1998.

[24] F. Reichmeyer, L. Ong, A. Terzis, L. Zhang, and R. Yavatkar, "A two-tier resource management model for differentiated services networks," Internet Draft, Internet Engineering Task Force, Nov. 1998. Work in progress.

[25] D. Awduche, L. Berger, D. Gan, T. Li, G. Swallow, and V. Srinivasan, "Extensions to RSVP for LSP tunnels," Internet Draft, Internet Engineering Task Force, Mar. 1999. Work in progress.

[26] R. Guerin, S. Herzog, and S. Blake, "Aggregating RSVP-based QoS requests," Internet Draft, Internet Engineering Task Force, Nov. 1997. Work in progress.

[27] F. Baker, "Aggregation of RSVP for IP4 and IP6 reservations," Internet Draft, Internet Engineering Task Force, June 1999. Work in progress.

[28] G. Feher, K. Nemeth, M. Maliosz, I. Cselenyi, J. Bergkvist, D. Ahlard, and T. Engborg, "Boomerang - a simple protocol for resource reservation in ip networks," in IEEE Workshop on QoS Support for Real-Time Internet Applications, (Vancouver, Canada), June 1999.

[29] P. Pan, E. Hahne, and H. Schulzrinne, "BGRP: A Framework for Scalable Resource Reservation," Internet Draft, Internet Engineering Task Force, Jan. 2000. Work in progress. 


\begin{tabular}{|c|c||r|r||r|}
\hline $\begin{array}{c}\text { Time } \\
\text { Interval }\end{array}$ & Region Granularity & $\begin{array}{r}\text { \# Source-Dest. Pairs } \\
\text { (\# RSVP Reservations) }\end{array}$ & $\begin{array}{r}\text { \# Destinations } \\
\text { (\# BGRP Res'ns) }\end{array}$ & Gain \\
\hline \hline \multirow{3}{9}{90 sec. } & $\begin{array}{c}\text { Application (Source = Address + Port ; } \\
\text { Destination = Address + Port + Protocol) }\end{array}$ & 339,245 & 208,559 & 1.6 \\
\cline { 2 - 5 } & Host (IP Address) & 131,009 & 40,538 & 3.2 \\
\cline { 2 - 5 } & Network (CIDR Prefix) & 79,786 & 20,887 & 3.8 \\
\cline { 2 - 5 } & Domain (AS) & 20,857 & 2,891 & 7.2 \\
\hline \hline 1 month & Domain (AS) & $7,900,362$ & 5,001 & $1,579.8$ \\
\hline
\end{tabular}

Table 1: Number of aggregate flows seen in packet traces 


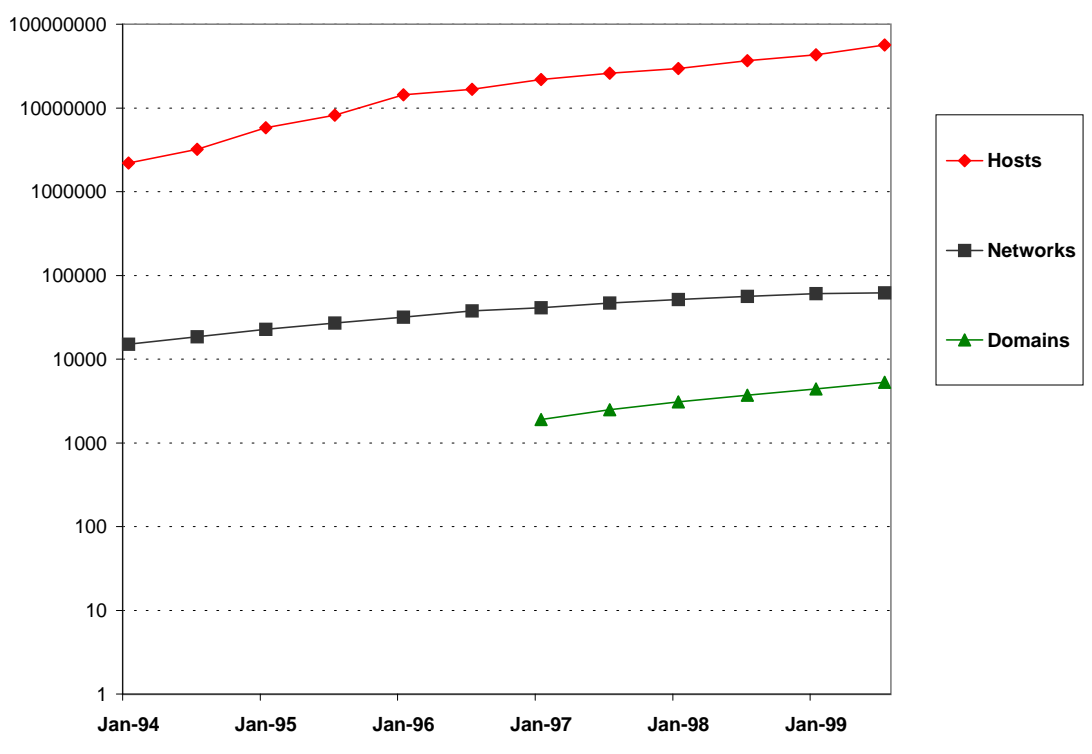

Figure 1: The growth of the Internet from 1994 to 1999 [7, 8, 9]. 


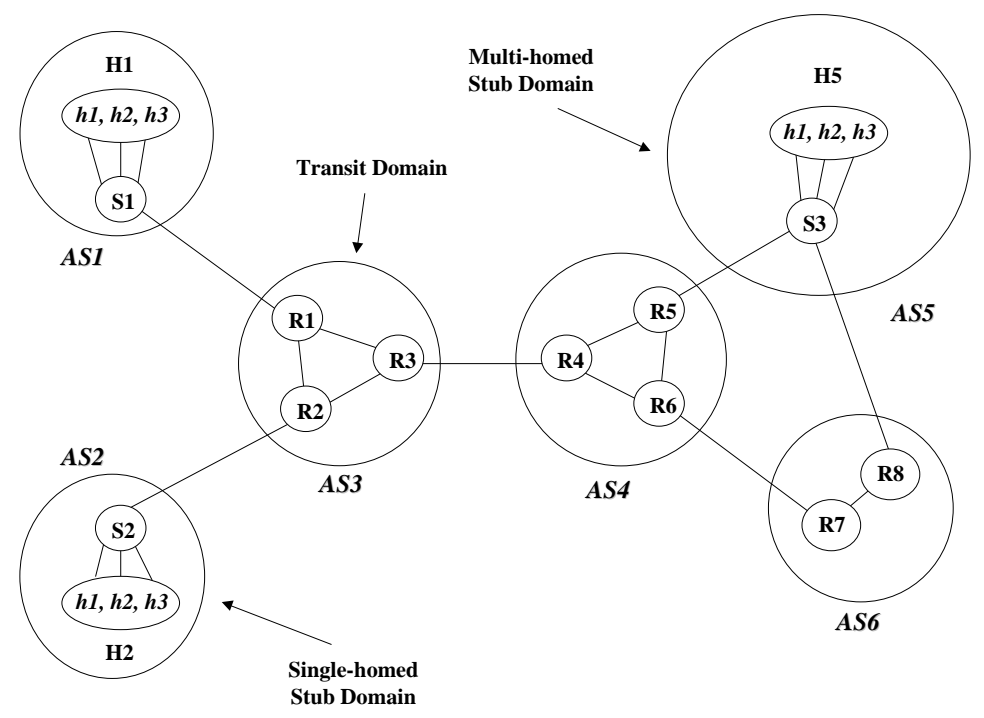

Figure 2: Example of Internet domains. There are two types of stub domains: single-homed stub domains connect to the backbone at a single point, multi-homed stub domains at several points. 


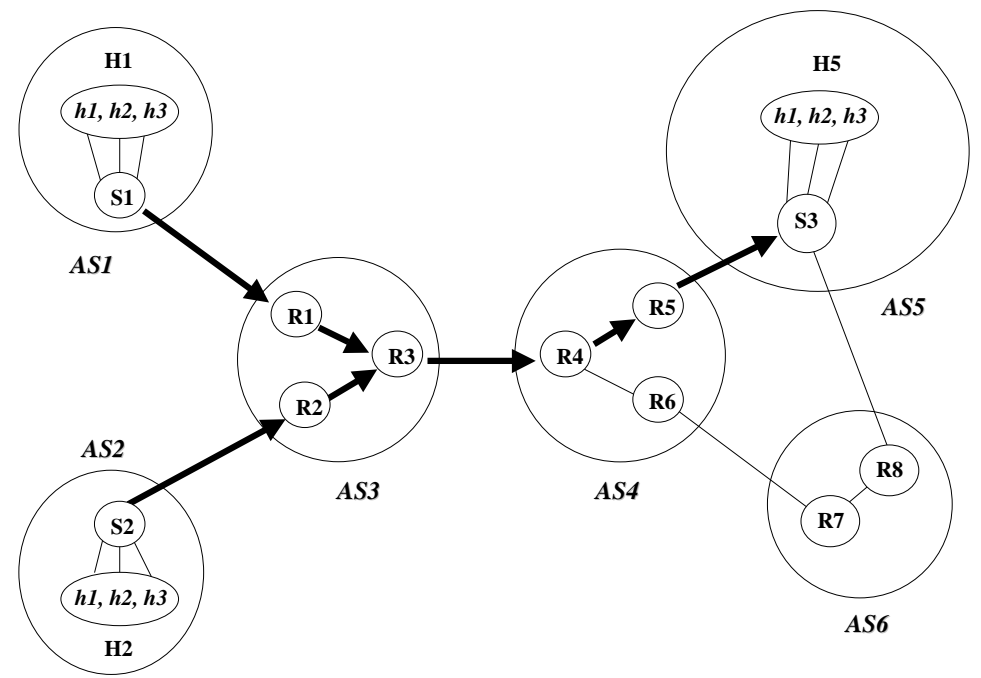

Figure 3: Example of a sink tree rooted at $S_{3}$ 


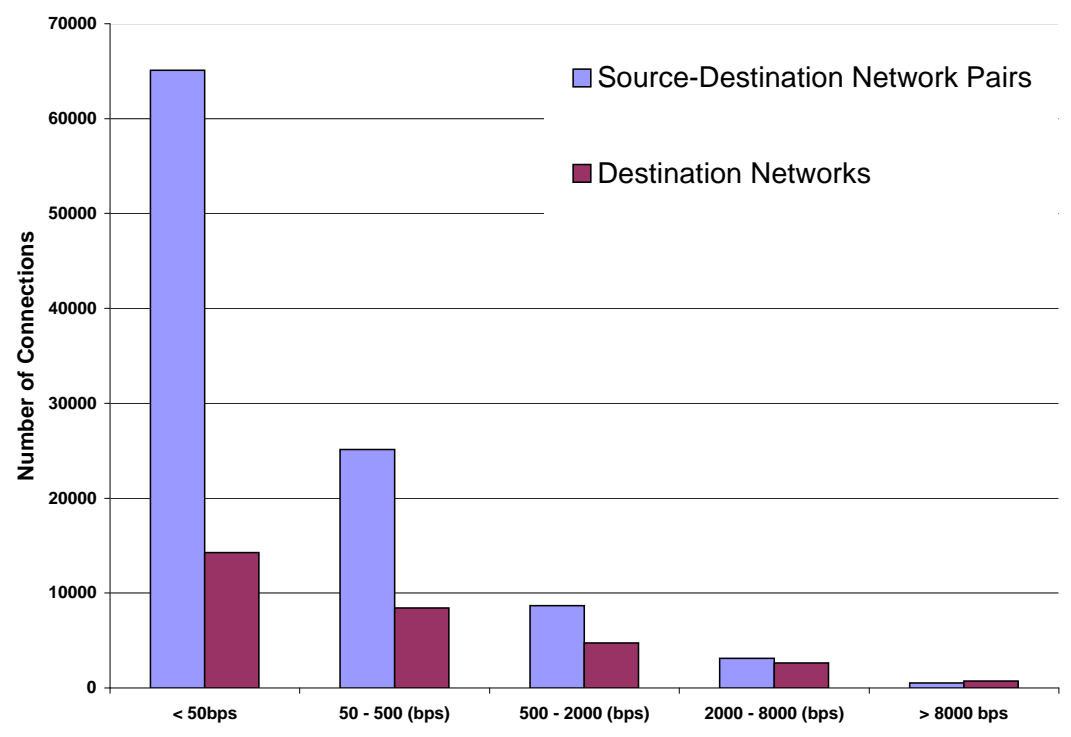

(a) Number of reservations, broken down by bandwidth

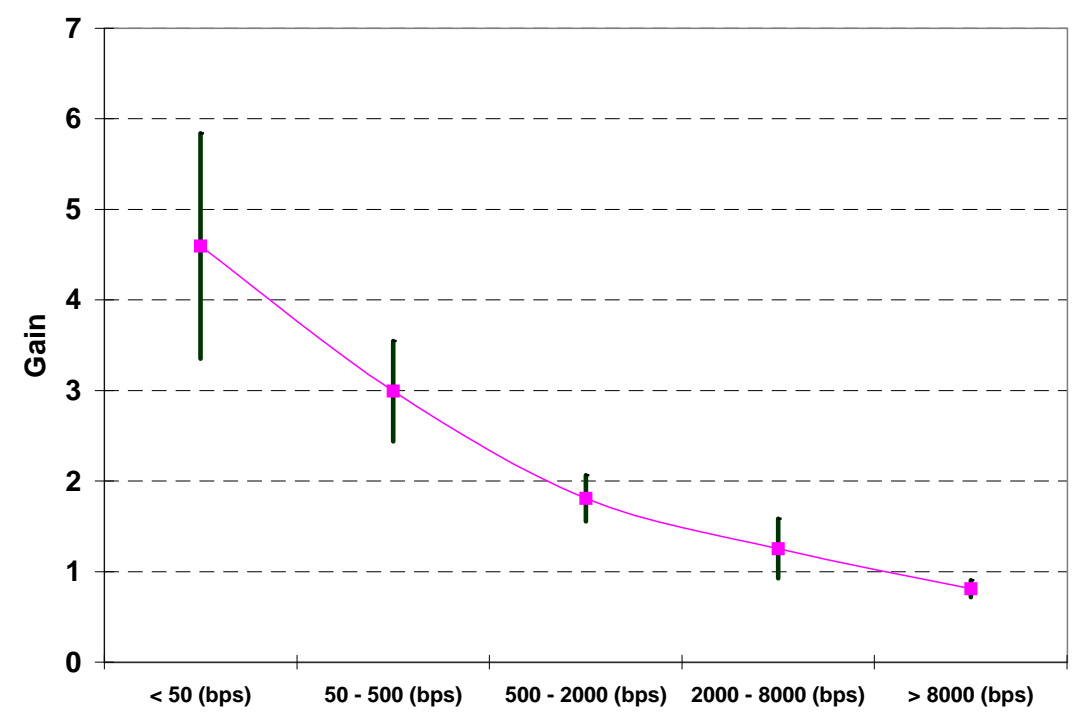

(b) Gain for each bandwidth class

Figure 4: Distribution of reservations by bandwidth 


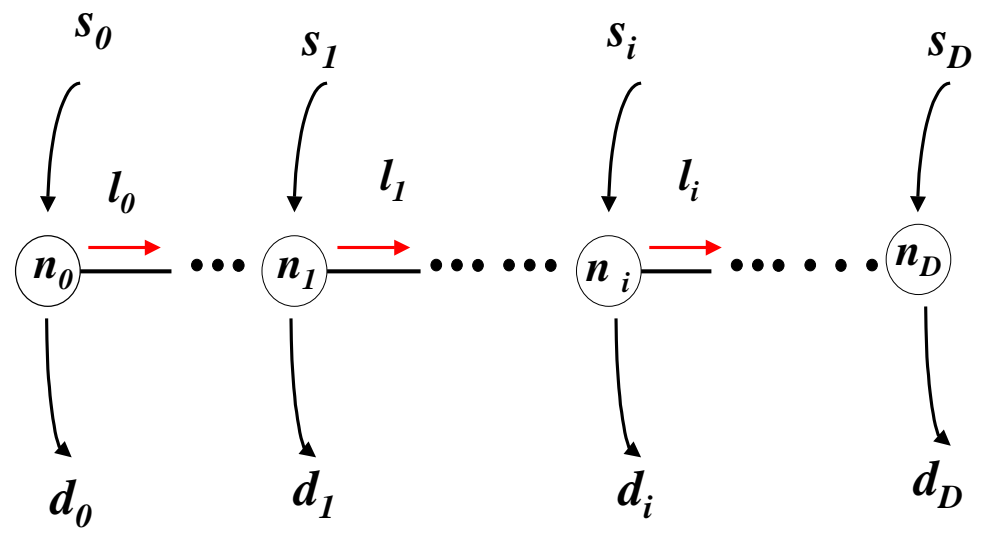

Figure 5: Model for analyzing the topological distribution of demand 


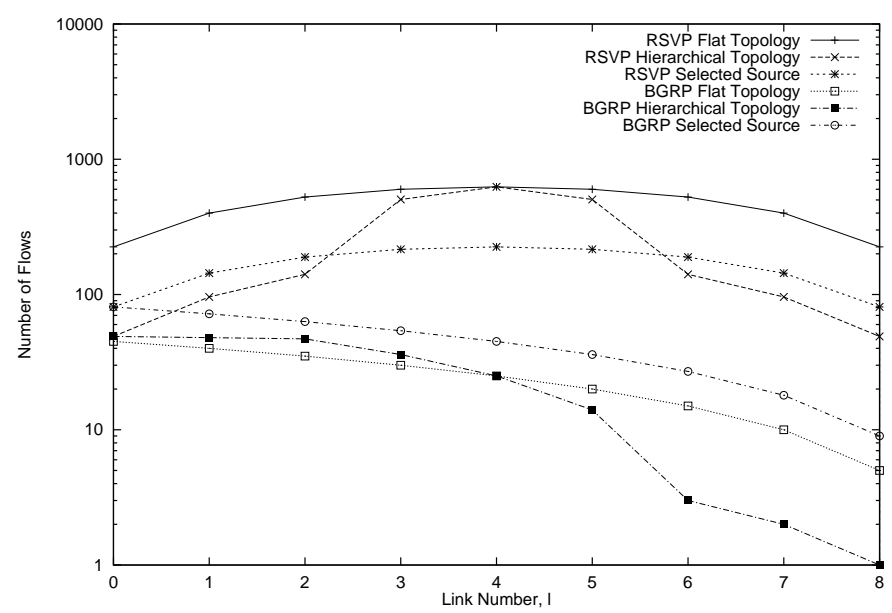

(a) Number of reservations per link

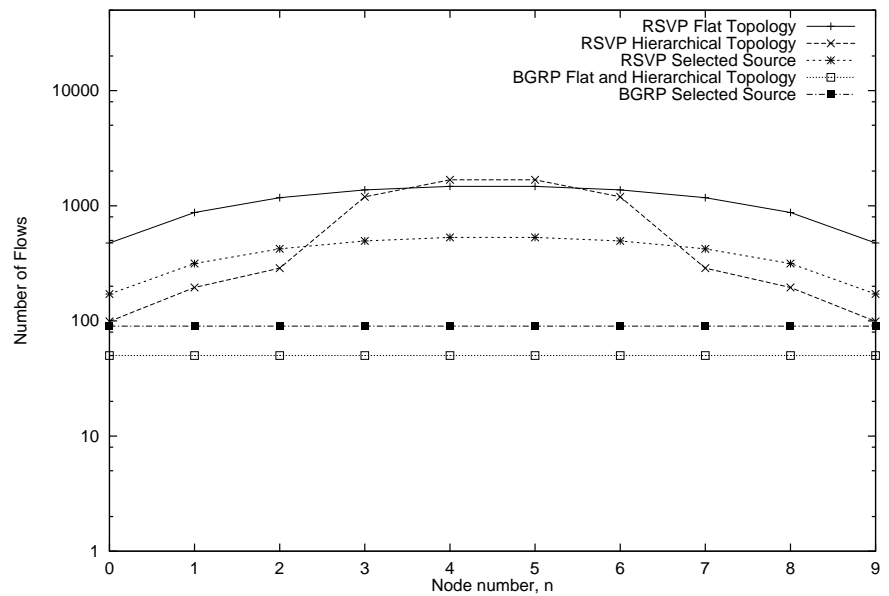

(b) Number of reservations per node

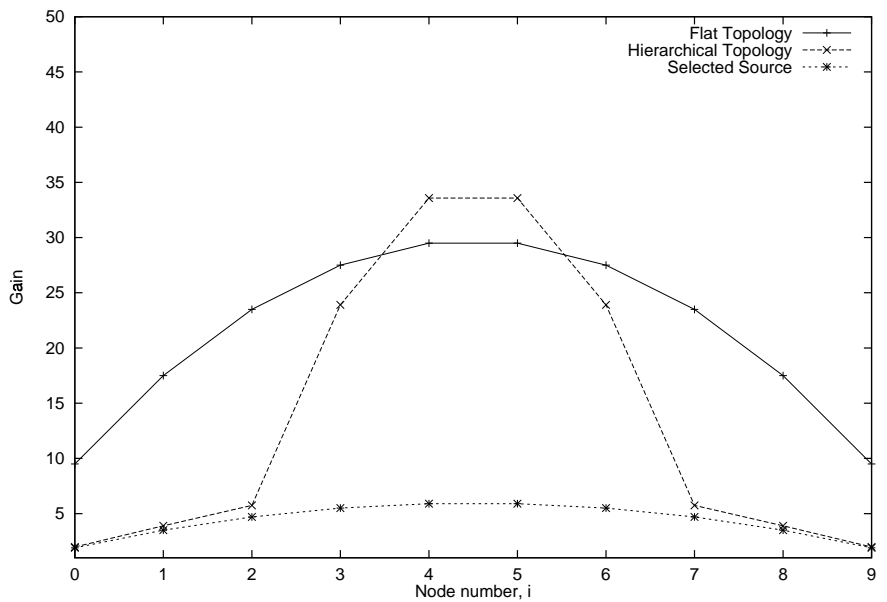

(c) The gain, $N_{i}^{\mathrm{RSVP}} / N_{i}^{\mathrm{BGRP}}$

Figure 6: Worst case scaling comparison between RSVP and BGRP. 


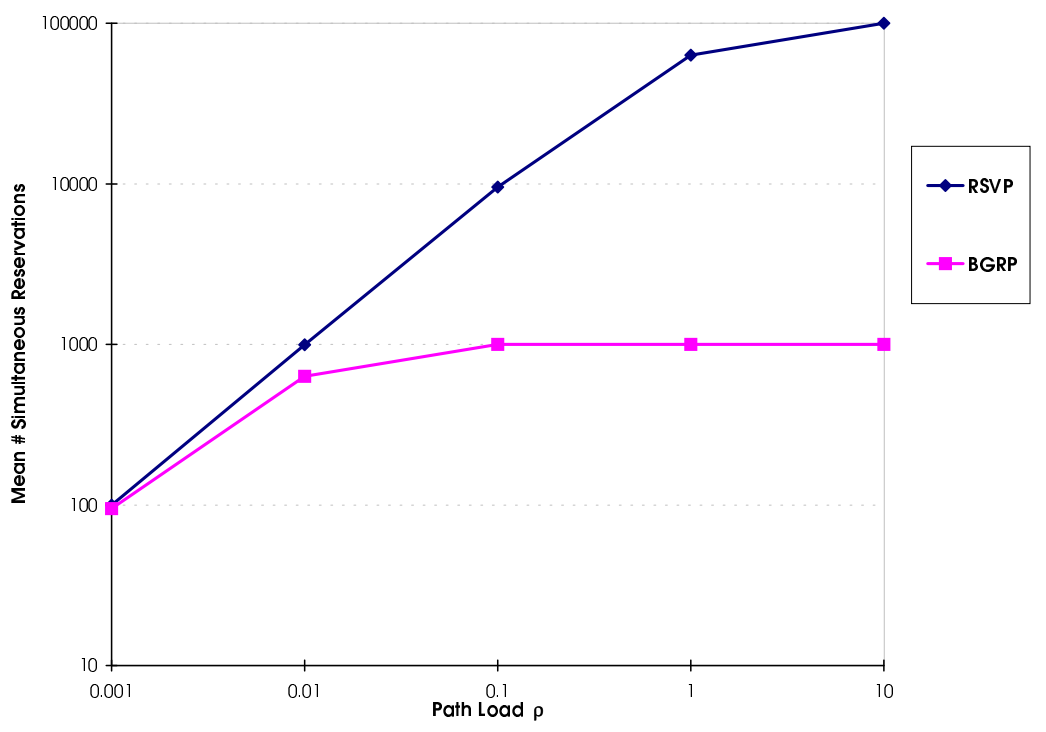

Figure 7: BGRP and RSVP reservation counts as functions of load $\rho$ 


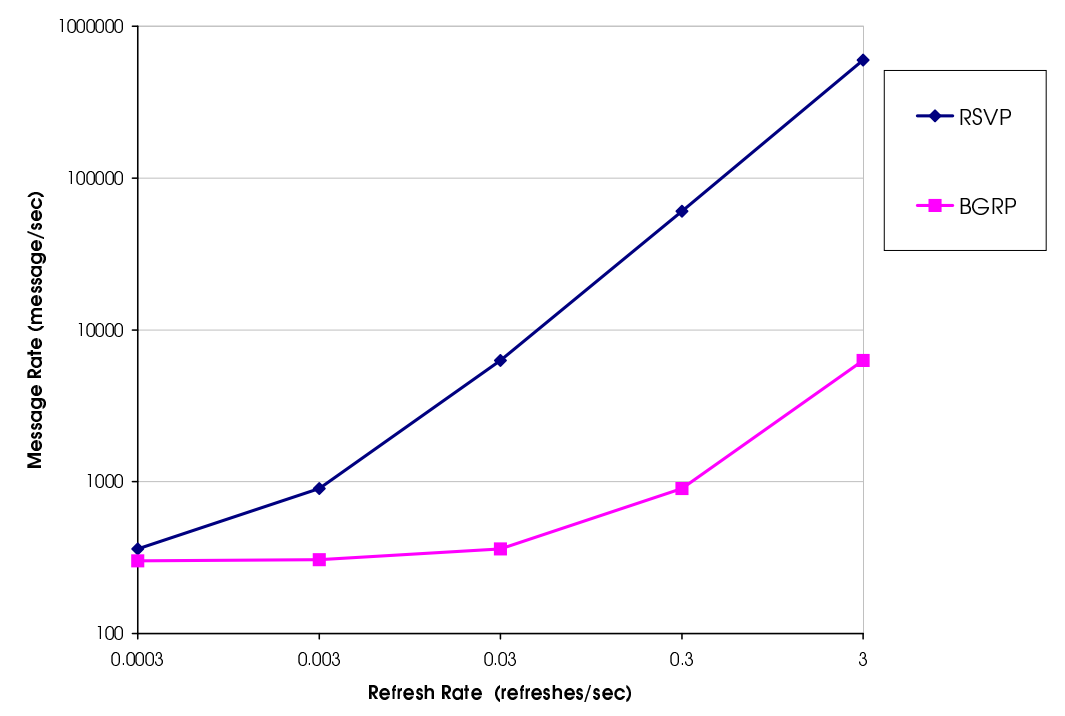

Figure 8: BGRP and RSVP message rates as functions of refresh rate $\eta$ 


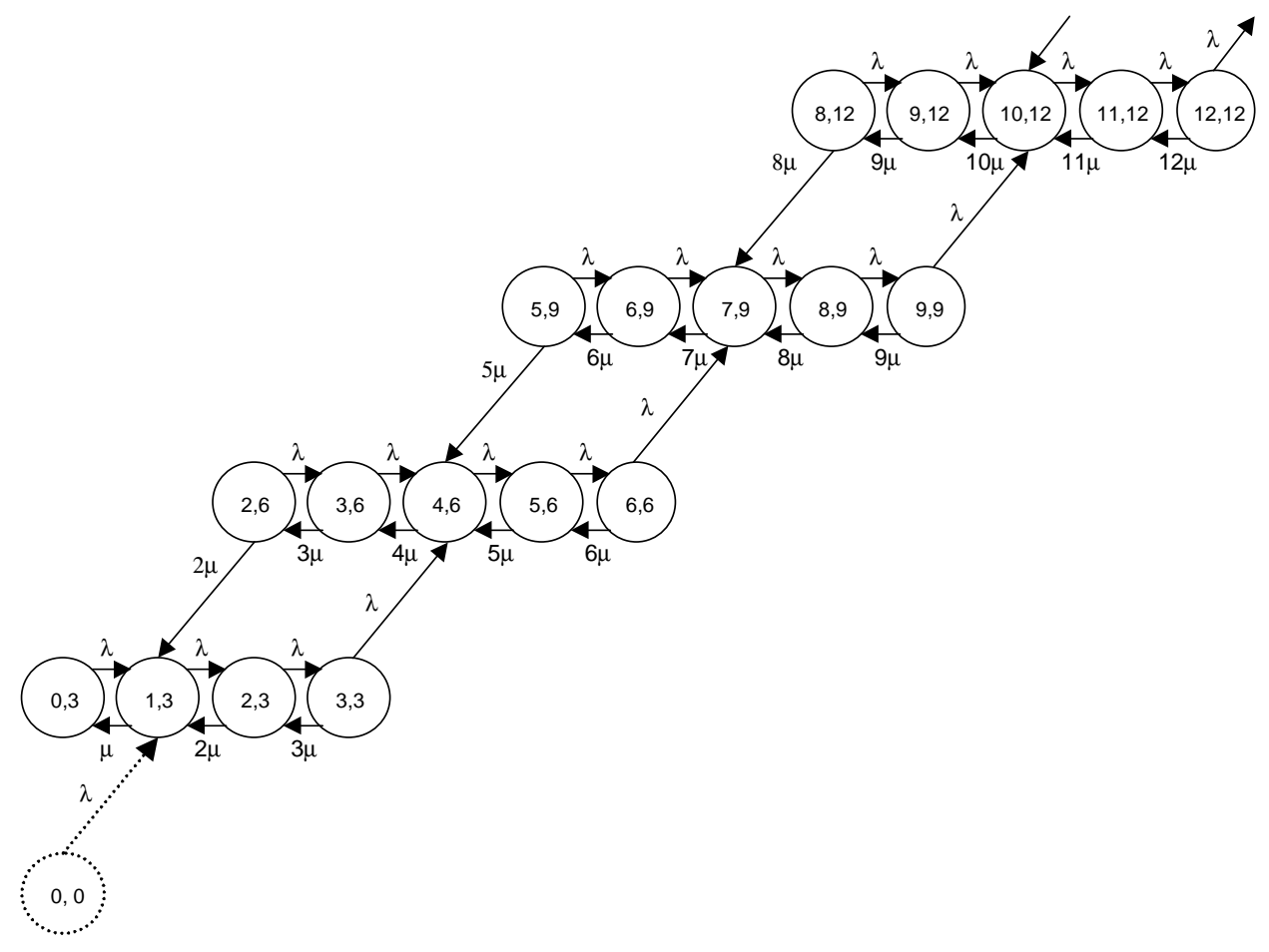

Figure 9: State transition diagram for $Q=3$ 


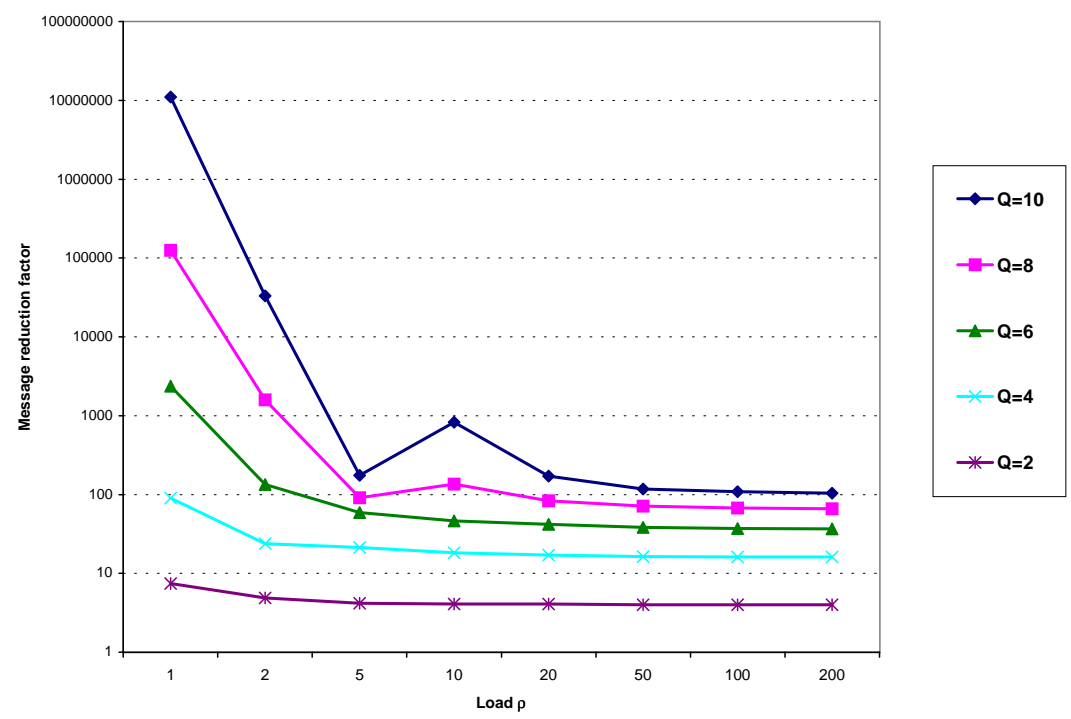

Figure 10: Message reduction factor as function of $Q$ and $\rho$ 